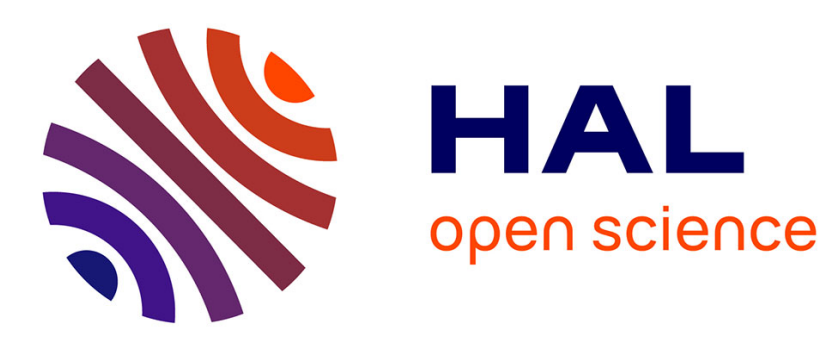

\title{
Nonlinear forced vibration of damped plates by an asymptotic numerical method
}

Farid Boumédiène, A. Miloudi, Jean-Marc Cadou, L. Duigou, E.H Boutyour

\section{To cite this version:}

Farid Boumédiène, A. Miloudi, Jean-Marc Cadou, L. Duigou, E.H Boutyour. Nonlinear forced vibration of damped plates by an asymptotic numerical method. Computers \& Structures, 2009, 87 (23-24), pp.1508-1515. 10.1016/j.compstruc.2009.07.005 . hal-00494489

\section{HAL Id: hal-00494489 \\ https://hal.science/hal-00494489}

Submitted on 20 Jun 2018

HAL is a multi-disciplinary open access archive for the deposit and dissemination of scientific research documents, whether they are published or not. The documents may come from teaching and research institutions in France or abroad, or from public or private research centers.
L'archive ouverte pluridisciplinaire HAL, est destinée au dépôt et à la diffusion de documents scientifiques de niveau recherche, publiés ou non, émanant des établissements d'enseignement et de recherche français ou étrangers, des laboratoires publics ou privés. 


\title{
Nonlinear forced vibration of damped plates by an asymptotic numerical method
}

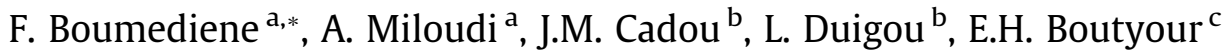 \\ a Laboratoire de Mécanique Avancée, Faculté de Génie Mécanique \& Génie des Procédés, USTHB, BP 32, El Alia, 16111 Bab Ezzouar, Alger, Algeria \\ ${ }^{\mathrm{b}}$ Laboratoire d'Ingénierie des Matériaux de Bretagne, Université Européenne de Bretagne, Université de Bretagne Sud, Rue de Saint Maudé, BP 92116,56321 Lorient Cedex, France \\ 'Département de Physique Appliquée, Faculté des Sciences et Techniques, Université Hassan I, BP 577, Settat, Morocco
}

\begin{abstract}
This work deals with damped nonlinear forced vibrations of thin elastic rectangular plates subjected to harmonic excitation by an asymptotic numerical method. Using the harmonic balance method and Hamilton's principle, the governing equation is converted into a static formulation. A mixed formulation is used to transform the problem from cubic nonlinearity to quadratic one sequence. Displacement, stress and frequency are represented by power series with respect to a path parameter. Equating the like powers of this parameter, the nonlinear governing equation is transformed into a sequence of linear problems with the same stiffness matrix. Through a single matrix inversion, a considerable number of terms of the perturbation series can easily be computed with a limited computation time. The starting point, corresponding to a regular solution, is obtained by the Newton-Raphson method. In order to increase the step length, Padé approximants are used. Numerical tests are presented and compared with numerical and analytical results in the literature, for different boundary conditions, excitations and damping coefficients.
\end{abstract}

\section{Introduction}

To minimize the structural weight, thin plates are widely used in many industrial fields such as aeronautic, mechanics and civil engineering. These structures are often forced into high-amplitude vibrations, inducing significant geometrical nonlinearity, which is a source of complex instability phenomena. To reduce the effect of such problems, a common approach consists in introducing a damping component into the structure. But the principal difficulty, in the analysis of such problems, lies in the presence, on the one hand, of nonlinearity, source of complex phenomena such as bifurcations, jumping and chaos, and on the other hand of damping, which generally leads to complex solutions.

In the literature, most investigations which take damping into account are limited to linear vibrations, leading to a complex eigenvalue problem. Only a few works combine both geometrical nonlinearity and damping: Amabili made an experimental and numerical study of plates with viscous damping and subjected to harmonic excitation $[1,2]$. This author studied also numerically circular cylindrical panels with viscous damping [3]. Ganapathi et al. determined the loss factor of a sandwich beam with a viscoelastic central layer, using the finite element method and an iterative approach [4]. An amplitude equation, based on an approximated harmonic balance method and Galerkin's procedure, was proposed by

\footnotetext{
* Corresponding author.

E-mail address: boumedienefaiza@yahoo.fr (F. Boumediene).
}

Daya et al. [5] to study sandwich beams and plates with central viscoelastic layers. The same approach was extended to the analysis of circular sandwich rings by Boutyour et al. [6]. Touzé and Amabili built reduced-order models for damped geometrically nonlinear systems [7]. They considered a modal viscous damping. Ribeiro and Petyt [8-10] used the hierarchical finite element method in an in-depth investigation of the nonlinear response of clamped rectangular plates. In these last papers, the authors considered mass proportional hysteretic damping. A similar approach was used by Ribeiro and Petyt to investigate the forced response of simply-supported plates with immovable edges [11] and of beams and plates, by following the thin and thick theories [12]. In these last articles, the authors considered stiffness proportional damping.

This paper deals with the nonlinear vibration of damped plates by an asymptotic numerical method (ANM). In previous works using this method $[13,14]$ the damping is generally neglected. In our paper, the damping is taken into account in a simple and classical manner type Rayleigh. Even if this structural damping is relatively simple, it represents a large part of engineering applications. The asymptotic numerical method (ANM) is used in its last efficient version based on a continuation method with use of Padé approximants [15].

As it is well known, in the investigation of thin plates' behavior, one assumes that the rotations are moderate and the use of von Karman model was validated in several previous referenced works $[1,11,14]$. 
Using this theory, the harmonic balance method and Hamilton's principle, the initial governing motion equation is converted into an operational form.

Coupling a perturbation technique and the finite element method, the nonlinear problem is transformed into a sequence of algebraic linear problems. Padé approximant method was used [15] to increase the step length and so to reduce computation time. The aim of this method is to improve the continuation method by replacing the polynomial approximation by a rational one. The obtained results are compared with the literature and with analytical approaches. To show the effectiveness of the proposed algorithm, several examples of rectangular plates, with various boundary conditions and excitations, are presented.

\section{Mathematical formulation}

Let us consider a thin rectangular plate with a coordinate system $(O ; x, y, z)$, the origin $O$ being situated at one corner. The displacement components of the plate's middle surface are denoted by $u, v$ and $w$, where $u$ and $v$ are the in-plane displacements and $w$ the transverse displacement in the $x, y$ and $z$ directions, respectively. The associated Green-Lagrange strain varies linearly with respect to the thickness:

$\boldsymbol{\varepsilon}=\gamma+z \boldsymbol{k}$

where $\gamma=\gamma^{\boldsymbol{l}}+\gamma^{\boldsymbol{n} \boldsymbol{l}}$ is the generalized membrane strain which can be broken down into a linear and nonlinear part, and $\boldsymbol{k}$ is the bending strain.

The nonlinear strain-displacement relationships associated with the von-Karman plate theory are given by:

$$
\begin{aligned}
& \boldsymbol{\gamma}^{\boldsymbol{l}}=\left\{\begin{array}{c}
\partial u / \partial x \\
\partial v / \partial y \\
\partial u / \partial y+\partial v / \partial x
\end{array}\right\}, \quad \gamma^{\boldsymbol{n} \boldsymbol{l}}=\frac{1}{2}\left\{\begin{array}{c}
(\partial w / \partial x)^{2} \\
(\partial w / \partial y)^{2} \\
2(\partial w / \partial x)(\partial w / \partial y)
\end{array}\right\}, \\
& \boldsymbol{k}=-\left\{\begin{array}{c}
\partial^{2} w / \partial x^{2} \\
\partial^{2} w / \partial y^{2} \\
2 \partial^{2} w / \partial x \partial y
\end{array}\right\}
\end{aligned}
$$

The strain can be written by an operator notation as follow:

$\boldsymbol{\varepsilon}=\left[\boldsymbol{B}_{\boldsymbol{l}}+\frac{1}{2} \boldsymbol{B}_{\boldsymbol{n} \boldsymbol{l}}(\boldsymbol{U})\right] \boldsymbol{U}$

where $\boldsymbol{U}$ is the displacement vector given by $\boldsymbol{U}=\{u, v, w\}^{t} ; \boldsymbol{B}_{\boldsymbol{l}}$ and $\boldsymbol{B}_{\boldsymbol{n} \mathbf{l}}$ are the linear and nonlinear strain-displacement operators, respectively. In our case: $\boldsymbol{B}_{\boldsymbol{l}} \boldsymbol{U}=\boldsymbol{\gamma}^{\boldsymbol{l}}+z \boldsymbol{k}$ and $\frac{1}{2} \boldsymbol{B}_{\boldsymbol{n l}}(\boldsymbol{U}) \boldsymbol{U}=\boldsymbol{\gamma}^{\boldsymbol{n l}}$.

The generalized stresses $\boldsymbol{N}$ are related to the strain for homogeneous and isotropic material by:

$N=\left\{\begin{array}{c}N_{x} \\ N_{y} \\ N_{x y}\end{array}\right\}=D \varepsilon$

where $\boldsymbol{D}$ is a symmetrical matrix containing material properties.

Neglecting the rotary inertia terms, the kinetic energy is given by:

$T=\frac{1}{2} \int_{S_{0}} \rho h \dot{\boldsymbol{U}}^{2} d S$

where $S_{0}$ is the plate's middle surface, $\rho$ is a density, $h$ is the plate's thickness and the dot is a derivation with respect to the time ' $t$ '.

Neglecting transverse stress $\sigma_{z}$ under Kirchhoff's hypotheses, the elastic strain energy $V$ of a plate is given by:

$V=\frac{1}{2} \int_{S_{0}} \boldsymbol{N} \boldsymbol{\varepsilon} d S$
Injecting expressions (1)-(4) into (6), taking into account the damping coefficients and using Hamilton's principle, the governing equation is obtained in the following form:

$$
\left\{\begin{array}{l}
\int_{S_{0}}^{t}\left[\boldsymbol{B}_{\boldsymbol{l}}+\boldsymbol{B}_{n \boldsymbol{l}}(\boldsymbol{U})\right] \boldsymbol{N} d S+\boldsymbol{C} \dot{U}-\boldsymbol{M} \ddot{U}=\boldsymbol{F} \\
\boldsymbol{N}=\boldsymbol{D}\left[\boldsymbol{B}_{\boldsymbol{l}}+\frac{1}{2} \boldsymbol{B}_{\boldsymbol{n} \boldsymbol{l}}(\boldsymbol{U})\right] \boldsymbol{U}
\end{array}\right.
$$

where $\boldsymbol{M}$ is the mass matrix, $\boldsymbol{F}$ is the external force vector and $\boldsymbol{C}$ is the viscous damping matrix of Rayleigh's type $\boldsymbol{C}=\alpha \boldsymbol{M}+\beta \boldsymbol{K}(\alpha$ and $\beta$ are two parameters and $\boldsymbol{K}$ is a stiffness matrix).

The problem is to solve the system (7) in which the unknowns are the displacement vectors, the stress vectors and the frequencies.

\section{Harmonic balance method}

The considered harmonic excitation is given by:

$\boldsymbol{F}(t)=\sum_{j=0}^{H-1}\left(\boldsymbol{F}_{c}^{j} \cos j \omega t+\boldsymbol{F}_{\boldsymbol{s}}^{\boldsymbol{j}} \sin j \omega t\right)$

where $H$ is the number of harmonics.

It is assumed that the response of the plate is harmonic and can be written down in the following form:

$$
\left\{\begin{array}{l}
\boldsymbol{U}(t)=\sum_{j=0}^{H-1} \boldsymbol{U}_{\boldsymbol{c}}^{j} \cos j \omega t+\boldsymbol{U}_{\boldsymbol{s}}^{\boldsymbol{j}} \sin j \omega t \\
\boldsymbol{N}(t)=\sum_{j=0}^{H-1} \boldsymbol{N}_{\boldsymbol{c}}^{j} \cos j \omega t+\boldsymbol{N}_{s}^{j} \sin j \omega t
\end{array}\right.
$$

New vectors $\overline{\boldsymbol{U}}$ and $\overline{\boldsymbol{N}}$ are introduced, containing all the harmonics and defined by:

$$
\left\{\begin{array}{l}
\bar{U}=\left[\boldsymbol{U}_{c}^{0}, \boldsymbol{U}_{c}^{1}, \boldsymbol{U}_{s}^{1}, \ldots, \boldsymbol{U}_{c}^{i}, \boldsymbol{U}_{s}^{i}, \ldots, \boldsymbol{U}_{c}^{\boldsymbol{H}-1}, \boldsymbol{U}_{s}^{\boldsymbol{H}-1}\right] \\
\overline{\boldsymbol{N}}=\left[\boldsymbol{N}_{c}^{0}, \boldsymbol{N}_{c}^{1}, \boldsymbol{N}_{\boldsymbol{s}}^{1}, \ldots, \boldsymbol{N}_{c}^{i}, \boldsymbol{N}_{\boldsymbol{s}}^{i}, \ldots, \boldsymbol{N}_{c}^{\boldsymbol{H}-1}, \boldsymbol{N}_{\boldsymbol{s}}^{\boldsymbol{H}-1}\right]
\end{array}\right.
$$

where " $c$ " denotes the co-sinus factor, "s" the sinus factor and $i$ the harmonic $i=0, H-1$.

By inserting series (9) and using the harmonic balance method, the system (7) is rewritten in the following form:

$$
\left\{\begin{array}{l}
\int_{S_{0}}^{t}\left[\overline{\boldsymbol{B}}_{\boldsymbol{l}}+\overline{\boldsymbol{B}}_{\boldsymbol{n} \boldsymbol{l}}(\overline{\boldsymbol{U}})\right] \overline{\boldsymbol{N}} d S+\omega \overline{\boldsymbol{C}} \overline{\boldsymbol{U}}-\omega^{2} \overline{\boldsymbol{M U}}=\overline{\boldsymbol{F}} \\
\overline{\boldsymbol{N}}=\overline{\mathbf{D}}\left[\overline{\boldsymbol{B}}_{\boldsymbol{l}}+\frac{\mathbf{1}}{\mathbf{2}} \overline{\boldsymbol{B}}_{\boldsymbol{n} \boldsymbol{l}}(\overline{\boldsymbol{U}})\right] \overline{\boldsymbol{U}}
\end{array}\right.
$$

where matrices $\overline{\boldsymbol{M}}, \overline{\boldsymbol{C}}, \overline{\mathbf{D}}, \overline{\boldsymbol{B}}_{\mathbf{l}}$ and $\overline{\boldsymbol{B}}_{n l}$ are derived from matrices $\boldsymbol{M}, \boldsymbol{C}$, $\boldsymbol{D}, \boldsymbol{F}, \boldsymbol{B}_{\boldsymbol{l}}$ and $\boldsymbol{B}_{\boldsymbol{n} \boldsymbol{l}}$, respectively (see Appendix A for a definition). The vector $\overline{\boldsymbol{F}}$ is derived from $\boldsymbol{F}$ and written in the form (10).

The last Eq. (11) is cubic with respect to the displacement and frequency, as our objective is to solve it using an asymptotic numerical method, it is written in a quadratic form with respect to an unknown vector $(\boldsymbol{\Lambda}, \omega, \Omega)$ :

$$
\begin{aligned}
& \langle\boldsymbol{L} \boldsymbol{\Lambda}, \boldsymbol{\delta} \boldsymbol{\Lambda}\rangle-\omega^{2}\langle\overline{\boldsymbol{M}} \boldsymbol{\Lambda}, \boldsymbol{\delta} \boldsymbol{\Lambda}\rangle+\Omega\langle\overline{\boldsymbol{C}} \boldsymbol{\Lambda}, \boldsymbol{\delta} \boldsymbol{\Lambda}\rangle+\langle\boldsymbol{Q}(\boldsymbol{\Lambda}, \boldsymbol{\Lambda}), \boldsymbol{\delta} \boldsymbol{\Lambda}\rangle \\
& \quad=\langle\overline{\boldsymbol{F}}, \boldsymbol{\delta} \boldsymbol{\Lambda}\rangle
\end{aligned}
$$

where $\boldsymbol{L}(\cdot)$ is a linear operator and $\boldsymbol{Q}(\cdot, \cdot)$ is a quadratic one defined by the following expressions:

$$
\begin{aligned}
& \langle\boldsymbol{L} \boldsymbol{\Lambda}, \boldsymbol{\delta} \boldsymbol{\Lambda}\rangle=\int_{S_{0}}^{t} \overline{\boldsymbol{B}}_{\boldsymbol{l}} \overline{\boldsymbol{N}} d S \\
& \langle\boldsymbol{Q}(\boldsymbol{\Lambda}, \boldsymbol{\Lambda}), \boldsymbol{\delta} \boldsymbol{\Lambda}\rangle=\int_{S_{0}}^{t} \overline{\boldsymbol{B}}_{\boldsymbol{n} \boldsymbol{l}}(\overline{\boldsymbol{U}}) \overline{\boldsymbol{N}} d S \\
& \boldsymbol{\Lambda}={ }^{t}[\overline{\boldsymbol{U}}, \overline{\boldsymbol{N}}] \text { is the mixed displacement-stress vector } \\
& \Omega=\omega^{2}
\end{aligned}
$$




\section{Asymptotic numerical method}

\subsection{Asymptotic expansion}

Let us consider a regular solution $\left(\boldsymbol{\Lambda}_{0}, \omega_{0}, \Omega_{0}\right)$ of the nonlinear problem (12), the basic idea of the ANM consists in searching for the solution path in the vicinity of this point, by power series with respect to a path parameter ' $a$ ':

$\left\{\begin{array}{l}\boldsymbol{\Lambda}(a)=\boldsymbol{\Lambda}_{0}+a \boldsymbol{\Lambda}_{1}+a^{2} \boldsymbol{\Lambda}_{2}+\cdots+a^{n} \boldsymbol{\Lambda}_{n} \\ \omega(a)=\omega_{0}+a \omega_{1}+a^{2} \omega_{2}+\cdots+a^{n} \omega_{n} \\ \Omega(a)=\Omega_{0}+a \Omega_{1}+a^{2} \Omega_{2}+\cdots+a^{n} \Omega_{n}\end{array}\right.$

where $\left(\boldsymbol{\Lambda}_{\boldsymbol{p}}, \omega_{p}, \Omega_{p}\right)$ is the new unknown parameter to be computed.

The path parameter used in the series (13) can be identified as the projection of the displacement increment $\left(\overline{\boldsymbol{U}}-\overline{\boldsymbol{U}}_{0}\right)$, and the frequency increment $\left(\omega-\omega_{0}\right)$, on the tangent vector $\left(\overline{\boldsymbol{U}}_{1}, \omega_{1}\right)$ :

$a=\left\langle\overline{\boldsymbol{U}}-\overline{\boldsymbol{U}}_{0}, \overline{\boldsymbol{U}}_{1}\right\rangle+\left(\omega-\omega_{0}\right) \omega_{1}$

where $\langle\cdot, \cdot\rangle$ designates the Euclidian scalar product.

Introducing expressions (13) into Eqs. (12) and (14) and equating like powers of ' $a$ ', one gets the following set of linear problems:

Order 1

$\left\{\begin{array}{l}\boldsymbol{L}_{\boldsymbol{t}}\left(\boldsymbol{\Lambda}_{1}\right)=\left(\Omega_{1} \overline{\boldsymbol{M}}-\omega_{1} \overline{\boldsymbol{C}}\right) \boldsymbol{\Lambda}_{0} \\ \left\langle\overline{\boldsymbol{U}}_{1}, \overline{\boldsymbol{U}}_{1}\right\rangle+\omega_{1}^{2}=1\end{array}\right.$

Order $p$

$\left\{\begin{array}{l}\boldsymbol{L}_{\boldsymbol{t}}\left(\boldsymbol{\Lambda}_{\boldsymbol{p}}\right)=\sum_{i=0}^{p-1}\left(\Omega_{p-i} \overline{\boldsymbol{M}}-\omega_{p-i} \overline{\boldsymbol{C}}\right) \boldsymbol{\Lambda}_{\boldsymbol{i}}-\sum_{i=1}^{p-1} \boldsymbol{Q}\left(\boldsymbol{\Lambda}_{\boldsymbol{i}}, \boldsymbol{\Lambda}_{\boldsymbol{p}}-\boldsymbol{i}\right) \\ \left\langle\overline{\boldsymbol{U}}_{1}, \overline{\boldsymbol{U}}_{\boldsymbol{p}}\right\rangle+\omega_{1} \omega_{p}=0\end{array}\right.$

We have:

$\boldsymbol{L}_{\boldsymbol{t}}(\cdot)=\boldsymbol{L}(\cdot)+2 \boldsymbol{Q}\left(\boldsymbol{\Lambda}_{0}, \cdot\right)+\omega_{0} \overline{\boldsymbol{C}}-\omega_{0}^{2} \overline{\boldsymbol{M}}$

\subsection{Discretization by finite element method}

As well known, in the literature of ANM, to solve the problems (15) and (16) by a classical finite element method, one returns to a displacement formulation using behavior law (11). So, after discretization, one gets:

Order 1

$$
\left\{\begin{array}{l}
{\left[\overline{\boldsymbol{K}}_{t}^{0}\right]\left\{\boldsymbol{q}_{1}\right\}=\omega_{1}\left[2 \omega_{0} \overline{\boldsymbol{M}}-\overline{\boldsymbol{C}}\right]\left\{\boldsymbol{q}_{0}\right\}} \\
\left\langle\boldsymbol{q}_{1}, \boldsymbol{q}_{1}\right\rangle+\omega_{1}^{2}=1 \\
\left\{\overline{\boldsymbol{N}}_{1}\right\}=[\overline{\boldsymbol{D}}]\left[\overline{\boldsymbol{B}}_{\boldsymbol{l}}+\overline{\boldsymbol{B}}_{\boldsymbol{n l}}\left(\boldsymbol{q}_{0}\right)\right]\left\{\boldsymbol{q}_{1}\right\} \\
\Omega_{1}=2 \omega_{0} \omega_{1}
\end{array}\right.
$$

Order $p$

$$
\left\{\begin{array}{l}
{\left[\overline{\boldsymbol{K}}_{t}^{0}\right]\left\{\boldsymbol{q}_{\boldsymbol{p}}\right\}=\omega_{p}\left[2 \omega_{0} \overline{\boldsymbol{M}}-\overline{\boldsymbol{C}}\right]\left\{\boldsymbol{q}_{0}\right\}+\left\{\boldsymbol{F}_{\boldsymbol{p}}^{\boldsymbol{n} l}\right\}} \\
\left\langle\mathbf{q}_{1}, \mathbf{q}_{\boldsymbol{p}}\right\rangle+\omega_{1} \omega_{p}=0 \\
\left\{\overline{\boldsymbol{N}}_{\boldsymbol{p}}\right\}=[\overline{\boldsymbol{D}}]\left[\overline{\boldsymbol{B}}_{\boldsymbol{l}}+\overline{\boldsymbol{B}}_{\boldsymbol{n l}}\left(\mathbf{q}_{0}\right)\right]\left\{\boldsymbol{q}_{\boldsymbol{p}}\right\}+\underbrace{\frac{1}{2}[\overline{\boldsymbol{D}}] \sum_{i=1}^{p-i}\left[\overline{\boldsymbol{B}}^{\boldsymbol{n l}}\left(\boldsymbol{q}_{\boldsymbol{p}}-\boldsymbol{i}\right)\right]\left\{\boldsymbol{q}_{\boldsymbol{i}}\right\}}_{\overline{\boldsymbol{N}}_{\boldsymbol{p}}^{n l}} \\
\Omega_{p}=2 \omega_{0} \omega_{p}+\underbrace{\sum_{i=1}^{p-1} \omega_{i} \omega_{p-i}}_{\Omega_{p}^{n l}}
\end{array}\right.
$$

where $\left[\overline{\boldsymbol{K}}_{t}^{0}\right]$ denotes the tangent matrix at the starting point $\left(\boldsymbol{\Lambda}_{0}, \omega_{0}, \Omega_{0}\right)$ and $\left\{\boldsymbol{q}_{\boldsymbol{p}}\right\}$ is the discretized form of the displacement $\overline{\boldsymbol{U}}_{\boldsymbol{p}}$ and

$$
\begin{aligned}
\left\{\boldsymbol{F}_{\boldsymbol{p}}^{\boldsymbol{n l}}\right\}= & \Omega_{p}^{n l}[\overline{\boldsymbol{M}}]\left\{\boldsymbol{q}_{0}\right\}+\sum_{i=1}^{p-1}\left[\Omega_{p-i} \overline{\boldsymbol{M}}-\omega_{p-i} \overline{\boldsymbol{C}}\right]\left\{\boldsymbol{q}_{\boldsymbol{i}}\right\} \\
& -\int_{S_{0}}\left({ }^{t}\left[\overline{\boldsymbol{B}}_{\boldsymbol{l}}+\overline{\boldsymbol{B}}_{\boldsymbol{n} \boldsymbol{l}}\left(\boldsymbol{q}_{0}\right)\right]\left\{\overline{\boldsymbol{N}}_{\boldsymbol{p}}^{\boldsymbol{n} l}\right\}+\sum_{i=1}^{p-1}{ }^{t}\left[\overline{\boldsymbol{B}}_{\boldsymbol{n} \boldsymbol{l}}\left(\boldsymbol{q}_{\boldsymbol{i}}\right)\right]\left\{\overline{\boldsymbol{N}}_{\boldsymbol{p}}-\boldsymbol{i}\right\}\right) d S
\end{aligned}
$$

Note that the plates are modeled with the classical triangular shell elements DKT [16], with three nodes and six degrees of freedom per node $\left(u, v, w, \theta_{x}, \theta_{y}, \theta_{z}\right)$.

So, all unknown parameters of the series (13) can be determined by successively solving Eqs. (18) and (19) at each order $p$.

\subsection{Validity range and Padé approximants}

The polynomial solutions (13) coincide almost perfectly within the convergence radius, but they diverge out of this zone of validity. This limit can be computed automatically by using the following simple criterion proposed by Cochelin et al. [17]:

$a_{m s}=\left(\eta \frac{\left\|\mathbf{q}_{1}\right\|}{\left\|\mathbf{q}_{\boldsymbol{n}}\right\|}\right)^{1 /(n-1)}$

where $\eta$ is a small given number.

The polynomial representation (13) can be improved using rational fractions named Padé approximants [15]:

$$
\left\{\begin{array}{l}
\left\{\boldsymbol{q}^{p}(a)\right\}=\left\{\boldsymbol{q}_{0}\right\}+\sum_{i=1}^{n-1} f_{i}(a) a^{i}\left\{\boldsymbol{q}_{i}\right\} \\
\omega^{p}(a)=\omega_{0}+\sum_{i=1}^{n-1} f_{i}(a) a^{i} \omega_{i}
\end{array}\right.
$$

where $f_{i}(a)$ are rational fractions admitting the same denominator.

The validity range of the solution (21) is defined by the maximal value ' $a_{m p}$ ' of the path parameter " $a$ ". The relative difference between the displacements at two consecutive orders must be smaller than a given parameter $\mu$, which leads to:

$\mu \geq \frac{\left\|\boldsymbol{q}_{\boldsymbol{n}}\left(a_{m p}\right)-\boldsymbol{q}_{\boldsymbol{n}-1}\left(a_{m p}\right)\right\|}{\left\|\boldsymbol{q}_{\boldsymbol{n}}\left(a_{m p}\right)-\boldsymbol{q}_{0}\right\|}$

The iterative application of the ANM makes it possible to determine the whole of a complex nonlinear branch.

\section{Numerical results and validation}

In this part of our study, different examples of plates with various excitation types and boundary conditions are presented. Some of these examples are taken from the literature to validate our method. The material is aluminum with Young's modulus $E=70 \times 10^{9} \mathrm{~Pa}$, density $\rho=2778 \mathrm{~kg} / \mathrm{m}^{3}$ and Poisson's ratio $v=0.3$. The plates are modeled with DKT triangular shell elements with three nodes and six degrees of freedom per node $\left(u, v, w, \theta_{x}, \theta_{y}, \theta_{z}\right)$. For symmetry reasons, only a quarter of the plate has been discretized with 121 nodes (i.e. 726 dof for one harmonic). Based on previous studies, the accuracy parameter $\eta=10^{-4}$, the truncation order $n=15$ [18] and harmonics number $H=3$ [13] are adopted in the beginning for an automatic and optimal computational time. After that, an in-depth study is performed on the choice of optimal parameters.

\subsection{Conservative plate}

In order to validate our program, the nonlinear response of an undamped fully-clamped square plate is considered in Table 1. The length of the plate is equal to 240 times its thickness, the applied load is assumed to be non-dimensional with an amplitude $P_{0}^{d}=0.2$. Our results are extremely similar to the ones found in the literature $[9,14,19-21]$. 
Table 1

Frequency ratio $\omega / \omega_{l}$ of the nonlinear forced vibration of fully-clamped square plates under a harmonic uniform distributed force $\left(L / h=240, P_{0}^{d}=0.2^{\mathrm{a}}\right)$.

\begin{tabular}{|c|c|c|c|c|c|c|c|c|c|}
\hline \multicolumn{2}{|l|}{ ANM [14] } & \multicolumn{2}{|l|}{ HFEM [9] } & \multicolumn{4}{|c|}{ Other methods [19-21] } & \multicolumn{2}{|c|}{ Present results (ANM) } \\
\hline \multirow[t]{2}{*}{$\frac{W_{\max }}{h}$} & \multirow[t]{2}{*}{$\frac{\omega}{\omega_{l}}$} & \multirow[t]{2}{*}{$\frac{W_{\max }}{h}$} & \multirow[t]{2}{*}{$\frac{\omega}{\omega_{l}}$} & \multirow[t]{2}{*}{$\frac{W_{\max }}{h}$} & \multicolumn{3}{|l|}{$\omega / \omega_{l}$} & \multirow[t]{2}{*}{$\frac{W_{\max }}{h}$} & \multirow[t]{2}{*}{$\frac{\omega}{\omega_{l}}$} \\
\hline & & & & & FEM + LIN & Elliptic & Perturbation & & \\
\hline 0.2001 & 0.2160 & 0.2000 & 0.2432 & 0.2000 & 0.1033 & 0.1200 & 0.1227 & 0.2008 & 0.2444 \\
\hline-0.2000 & 1.4330 & -0.2072 & 1.4275 & -0.2000 & 1.4183 & 1.4195 & 1.4195 & -0.2062 & 1.4273 \\
\hline 0.4004 & 0.7532 & - & - & 0.4000 & 0.7372 & 0.7483 & 0.7484 & 0.4044 & 0.7532 \\
\hline-0.4001 & 1.2505 & - & - & -0.4000 & 1.2426 & 1.2490 & 1.2491 & -0.4012 & 1.2487 \\
\hline 0.6001 & 0.8949 & 0.6008 & 0.8971 & 0.6000 & 0.8746 & 0.8951 & 0.8956 & 0.6078 & 0.8887 \\
\hline-0.6001 & 1.2093 & -0.5901 & 1.2120 & -0.6000 & 1.1966 & 1.2117 & 1.2119 & -0.6006 & 1.2029 \\
\hline 0.8001 & 0.9912 & - & - & 0.8000 & 0.9617 & 0.9941 & 0.9954 & 0.8076 & 0.9773 \\
\hline-0.8001 & 1.2149 & - & - & -0.8000 & 1.1938 & 1.2203 & 1.2210 & -0.8009 & 1.2036 \\
\hline 1.0001 & 1.0769 & 1.0013 & 1.0803 & 1.000 & 1.0362 & 1.0822 & 1.0845 & 1.0032 & 1.0539 \\
\hline-1.0001 & 1.2457 & -0.9952 & 1.2475 & -1.000 & 1.2140 & 1.2540 & 1.2555 & -1.0029 & 1.2287 \\
\hline
\end{tabular}

${ }^{a}$ From Ref. [19] $P_{0}^{d}=c \cdot P^{d} / \rho h^{2} \omega_{l}^{2}, c=/ \int \phi d x d y / \int \phi^{2} d x d y ; \Phi$ : normalized mode shape; $P^{d}$ : amplitude of external distributed force (N/m ${ }^{2}$ ); $\rho$ is the density, $\omega_{l}$ is the linear frequency and $h$ is the thickness.

Table 2

Plates' characteristics.

\begin{tabular}{lllll}
\hline $\begin{array}{l}\text { Plate } \\
\text { number }\end{array}$ & $\begin{array}{l}\text { Length } L \\
(\mathrm{~m})\end{array}$ & $\begin{array}{l}\text { Width } l \\
(\mathrm{~m})\end{array}$ & $\begin{array}{l}\text { Thickness } h \\
(\mathrm{~m})\end{array}$ & $\begin{array}{l}\text { Damping } \\
\text { coefficients }\end{array}$ \\
\hline 1 & 0.6 & 0.3 & 0.001 & $\alpha=0, \beta$ \\
2 & 0.3 & 0.3 & 0.001 & $\alpha=0.065, \beta=0$ \\
\hline
\end{tabular}

\subsection{Damped plates}

In this section, two isotropic homogenous plates are analyzed. Their geometrical characteristics and the used damping coefficients are given in Table 2 . The first plate is rectangular with simply-supported boundary conditions and it is subjected to a vertically-distributed harmonic excitation. The second one is a square plate subjected to a vertically-distributed harmonic excitation. It is simply-supported with immovable edges [1]:

$$
\begin{cases}u=v=w=M_{x}=0 & \text { at } x=0, L \\ u=v=w=M_{y}=0 & \text { at } y=0, l\end{cases}
$$

where $M_{x}$ and $M_{y}$ are the bending moments per unit length according to $x$ and $y$ directions, respectively.

Fig. 1 shows the maximum vibration amplitudes of plate 1 , due to a harmonic distributed force with amplitudes of 5 and $10 \mathrm{~N} / \mathrm{m}^{2}$, and frequencies around the first resonance frequency. Considering the case of damping coefficients $\beta=10^{-3}$ and $\beta=10^{-4}$, it can be noted that the present approach gives the same results as those computed by HFEM and shooting methods [11]. For the second example, approximate functions [1] are used instead of the finite elements method to expand the middle surface displacements $u$, $v$ and $w$ :

$$
\left\{\begin{array}{l}
u(x, y, t)=\sum_{i=1}^{m} \sum_{j=1}^{n} u_{2 i, j}(t) \sin \left(\frac{2 \pi i}{l} x\right) \sin \left(\frac{\pi j}{L} y\right) \\
v(x, y, t)=\sum_{i=1}^{m} \sum_{j=1}^{n} v_{i, 2 j}(t) \sin \left(\frac{\pi i}{l} x\right) \sin \left(\frac{2 \pi j}{L} y\right) \\
w(x, y, t)=\sum_{i=1}^{\hat{m}} \sum_{j=1}^{\hat{n}} w_{i, j}(t) \sin \left(\frac{\pi i}{l} x\right) \sin \left(\frac{\pi j}{L} y\right)
\end{array}\right.
$$

The bending moments given in (23) can be written as:

$$
\begin{array}{ll}
M_{x}=\frac{E h^{3}}{12\left(1-v^{2}\right)}\left(\partial^{2} w / \partial x^{2}+v \partial^{2} w / \partial y^{2}\right)=0 & \text { at } x=0, L \\
M_{y}=\frac{E h^{3}}{12\left(1-v^{2}\right)}\left(\partial^{2} w / \partial y^{2}+v \partial^{2} w / \partial x^{2}\right)=0 & \text { at } y=0, l
\end{array}
$$

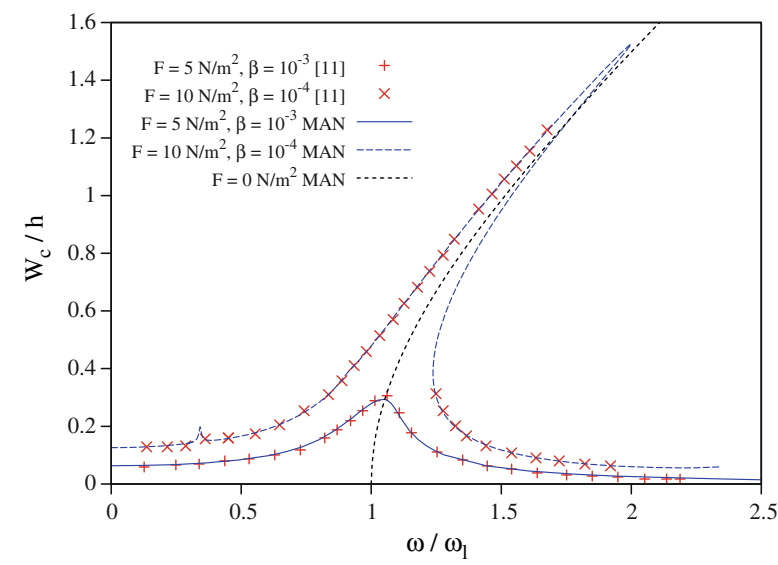

Fig. 1. The forced nonlinear vibration of damped rectangular plate no. 1 for various excitations and damping coefficients.

Therefore, the geometric boundary conditions associated to (23) are exactly satisfied by the expansions of $u, v$ and $w$ given by (24).

The asymptotic numerical method is applied to obtain the variation of displacement with respect to frequency. After that, the first generalized component of transversal displacement $w_{11}$ is compared with that given by previous works (Fig. 2a). It can be noted that our results coincide perfectly with those found in the literature $[1,22]$. On the basis of these analytical results, the transversal displacement of the plate centre is calculated. The plate centre displacements given by this approach (analytic + ANM) and by the finite element method (FEM + ANM) are compared in Fig. $2 b$.

In Figs. 3 and 4, the responses of fully-clamped plates (CC) and simply-supported plates (SS) are compared. As in the previous section, non-dimensional forces are adopted, and the free response is also computed using the asymptotic numerical method. It appears clearly that the simply-supported boundary conditions yield a larger nonlinear response than the clamped ones.

In addition to principal resonances, the present approach makes it possible to obtain higher harmonics resonances. Fig. 5 presents the responses of the structures with various harmonics numbers. In these tests, the adopted ANM parameters are: $n=20, \eta=10^{-4}$ and $\mu=10^{-4}$ (see Section 5.3 for more details about the choice of these parameters). It can be noted that with four harmonics, only one higher harmonic resonance is detected for a frequency $\omega \approx 0.3 \omega_{l}$, while the use of six harmonics gives two higher harmonic resonances, the first situated at $\omega \approx 0.2 \omega_{l}$, and the second at $\omega \approx 0.3 \omega_{l}$. Hence, to have a complete study of the geometrical 


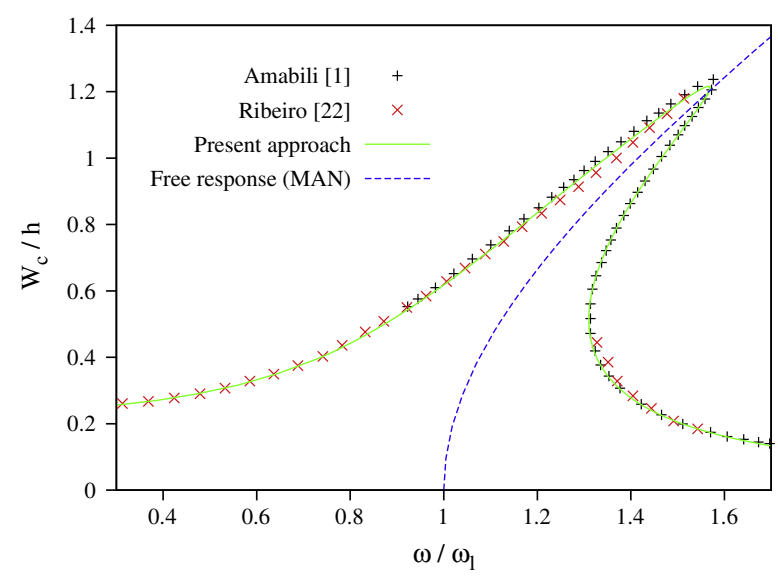

(a) The first generalized coordinate

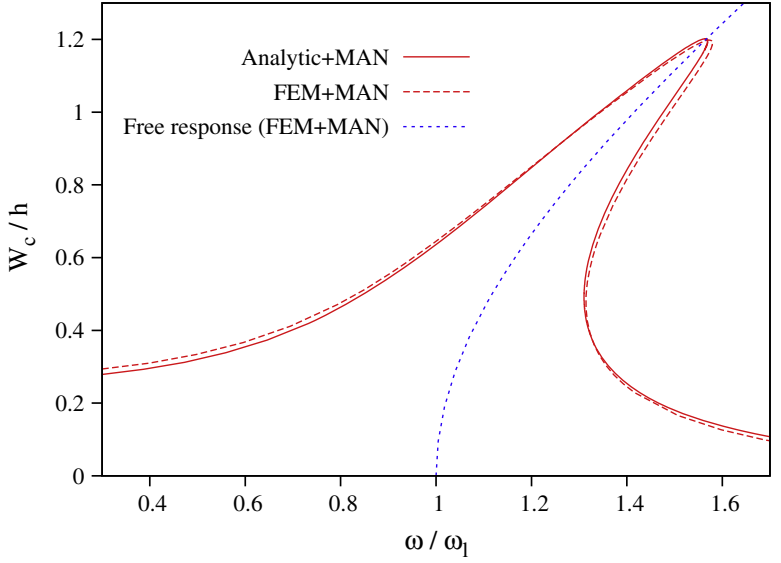

(b) Displacement of plate centre

Fig. 2. Forced nonlinear vibration of a damped square plate no. $2: F=1.74 \mathrm{~N}, \alpha=0.065, \beta=0$.

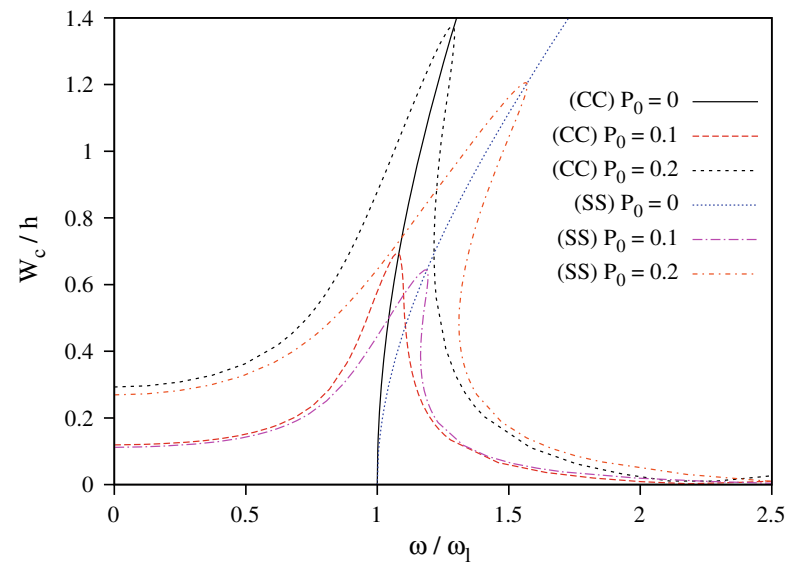

Fig. 3. Forced nonlinear vibration of damped square plate no. 1: $\alpha=2 * 0.065 * \omega_{l}$ and $\beta=0$.

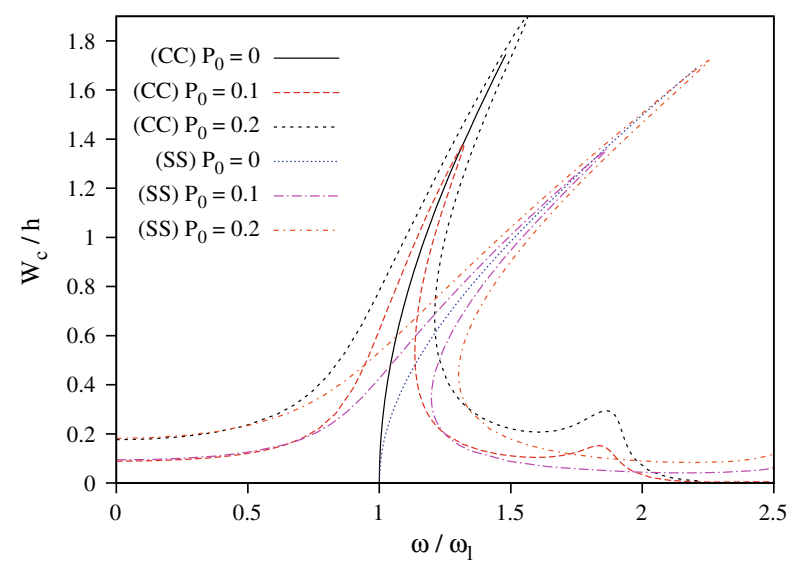

Fig. 4. Forced nonlinear vibration of damped rectangular plate no. 2 : $L / l=2$, $\beta=0.0001$ and $\alpha=0$.

nonlinear response, more harmonics are required. However, the computation time increases with the number of harmonics as detailed in the next section.

\subsection{Evaluation of computational cost}

For the first example, several tests were performed with different truncation orders, tolerance coefficients and excitation frequencies, so as to choose the adequate parameters. An excitation amplitude $F=40 \mathrm{~N} / \mathrm{m}^{2}$ and a damping factor of $\beta=10^{-4}$ were considered. The analysis was performed around the first mode $\left(0 \leqslant \omega \leqslant 2 \omega_{1}\right)$. In each test, one parameter was changed to see its influence on the solution's quality. The harmonic number $H=3$ was adopted in all these tests. Taking first the tolerance $\eta=10^{-4}$, and changing truncation order $n$ (Table 3 ), it can be noted that with the truncation order $n=20$, one gets smallest steps number and the better final residual. Then, for the second test, taking the truncation order $n=20$ and changing the tolerance $\eta$ (Table 4 ), it can be noted that with tolerance $\eta=10^{-3}$, one obtains the smallest steps number but a bad final residual. This tolerance can be taken into account and corrective steps added at the end of the computation. $\eta=10^{-4}$ was finally adopted for our tests.

Based on the results of these test, the following parameters of ANM are selected: truncation order $n=20$ and tolerance $\eta=10^{-4}$. The results obtained with the rational representation (the Padé approximants) for several values of the Padé tolerance parameter (Table 5) are then studied. Without Padé approximants, 67 steps have to be performed to have the entire curve from $\omega=0$ up to $\omega=2 \omega_{l}$. Note that with $\mu=10^{-3}$ the steps number is nearly half of polynomials one. Table 5 shows that the quality of the Padé approximants with $\mu=10^{-4}$ and $\mu=10^{-5}$ is really better than the polynomial.

Finally, the relative computational times, for different numbers of harmonics, are given in Table 6 . To obtain representative times, the considered number of nodes is equal to 435 (i.e. 2610 dof for one harmonic). This increases the number of terms in the matrix, and consequently the computational cost. In one step of the asymptotic numerical method, one has to perform only one matrix decomposition, to calculate all second members and to apply the Padé approximant. This last computation step does not require a great deal of computational time. For the matrix decomposition, the time increases with the harmonics number because the tangent matrix is constructed from $\left(2^{*} \mathrm{H}-1\right)^{2}$ blocs. In this study, a classical Crout's method is used. The relative CPU times are then defined as the ratio between the triangulation time of the tangent operator $\overline{\mathbf{K}}_{t}^{0}$ in nonlinear problems given in Eqs. (18) and (19), and the triangulation time of tangent operator $K$ in linear dynamical problems which contain only one harmonic: 


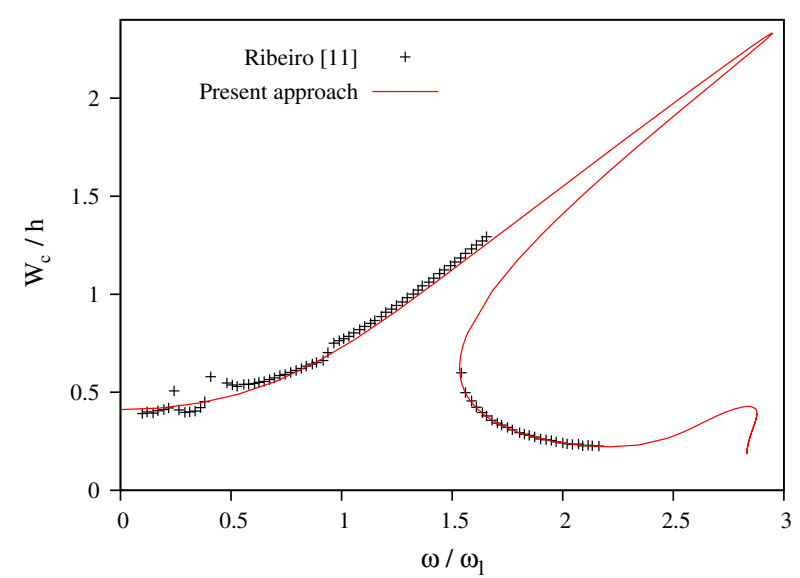

(a) Three harmonics $\mathrm{H}=3$

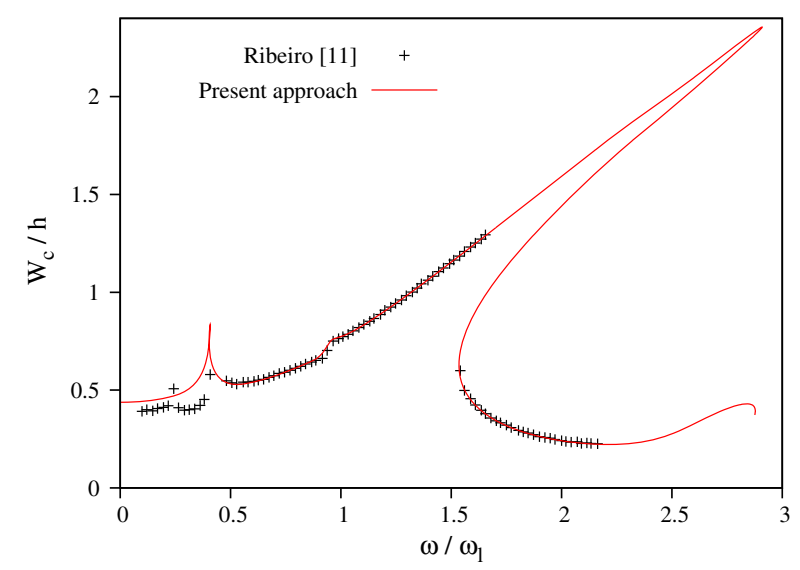

(c) Five harmonics $\mathrm{H}=5$

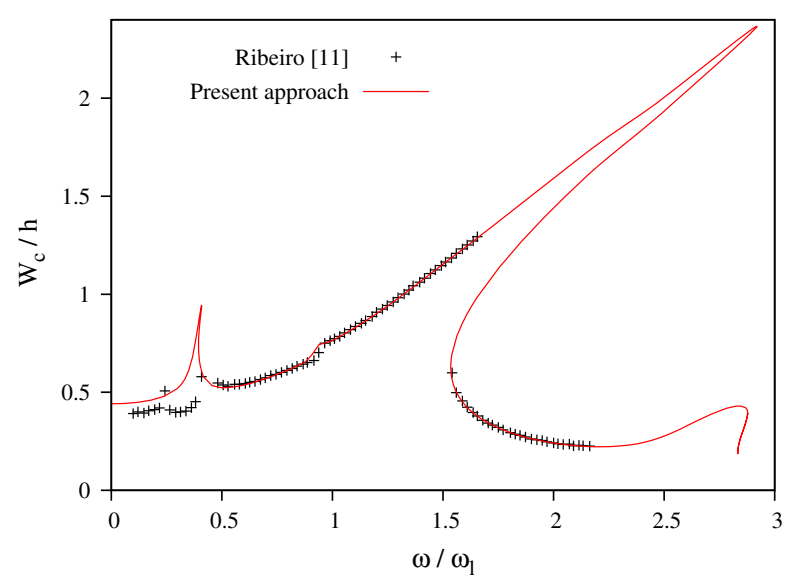

(b) Four harmonics $\mathrm{H}=4$

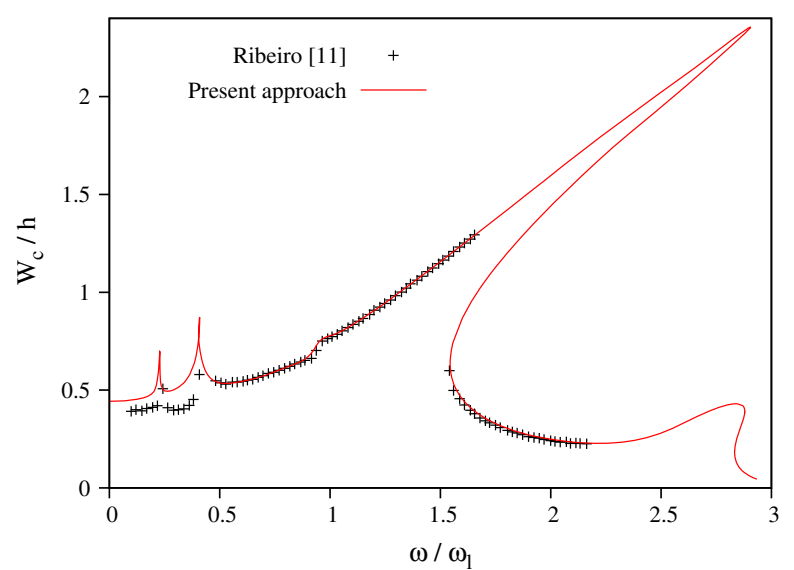

(d) Six haramonics $\mathrm{H}=6$

Fig. 5. Forced nonlinear vibration of damped rectangular plate no. 1: $F=40 \mathrm{~N} / \mathrm{m}^{2}, \alpha=0, \beta=0.001$.

Table 3

Step number and final residual of polynomial representation at $\omega=2 \omega_{1}$ with respect to the truncation number $n\left(\eta=10^{-4}, H=3\right)$.

\begin{tabular}{llll}
\hline Order $n$ & 19 & 20 & 21 \\
Step number & 70 & 67 & 75 \\
Final residual & 0.11 & 0.09 & 0.11 \\
\hline
\end{tabular}

\section{Table 4}

Step number and final residual of polynomial representation at $\omega=2 \omega_{1}$ with respect to tolerance $\eta(n=20, H=3)$.

\begin{tabular}{llll}
\hline Tolerance $\eta$ & $10^{-3}$ & $10^{-4}$ & $10^{-5}$ \\
Step number & 57 & 67 & 83 \\
Final residual & 1.045 & 0.090 & 0.004 \\
\hline
\end{tabular}

Table 5

Step number and final residual of the Padé representation at $\omega=2 \omega_{1}$ with respect to the Padé tolerance $\mu(n=20$ and $H=3)$.

\begin{tabular}{llll}
\hline Tolerance $\mu$ & $10^{-3}$ & $10^{-4}$ & $10^{-5}$ \\
Step number & 36 & 50 & 55 \\
Final residual & 0.1750 & 0.0151 & 0.0006 \\
\hline
\end{tabular}

$\mathrm{CPU}^{\text {relative }}=\frac{\mathrm{CPU}\left(\bar{K}_{t}^{0}\right)}{\operatorname{CPU}(K)}$

Table 6 shows that for six harmonics, the time of the nonlinear matrix decomposition is 127 times that required for the computation of the solution in a linear problem. This is very large time, but
Table 6

Relative CPU times versus the number of harmonics $H$.

\begin{tabular}{llllll}
\hline Harmonics number & 2 & 3 & 4 & 5 & 6 \\
CPU $^{\text {relative }}$ & 2.84 & 12.61 & 34.81 & 70.27 & 127.05 \\
\hline
\end{tabular}

this matrix decomposition must be also done with other methods (for example the Newton-Raphson). Furthermore, the NewtonRaphson method requires a great many steps [23] while our method allows us to obtain a large section of the solution with only one matrix decomposition.

The right hand side calculus time depends in one hand on the number of harmonics, and on the other hand of the truncation order. The ratio of the right hand side calculus time, with respect to the matrix decomposition time, is presented in Fig. 6. It can be noted that when the harmonics number is increased, the right hand side calculus time became more negligible compared to the decomposition time.

\section{Conclusion}

This paper develops an asymptotic numerical method to solve nonlinear forced vibration problems, taking into account structural damping of plates subjected to time-harmonic transversal excitation. A large part of the nonlinear solution is obtained by solving a sequence of linear problems having the same stiffness matrix. Iteration of this method, leading to a path-following technique, 


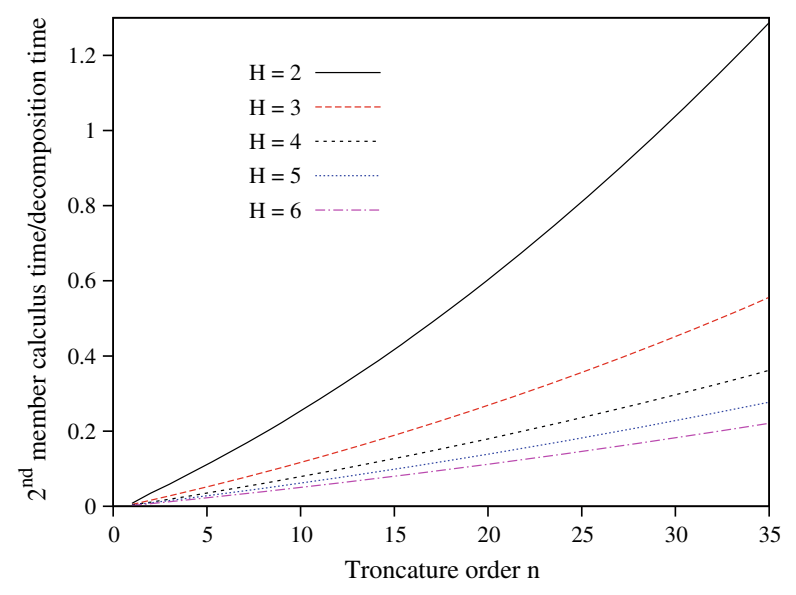

Fig. 6. Second member calculus time by decomposition time with respect to order number.

made it possible to obtain the nonlinear resonance curves at any desired range of amplitudes with the limit imposed by the von Karman theory. We reported numerical tests for forced vibrations of damped rectangular plates subjected to time-harmonic lateral excitations, and compared to calculations with numerical results available in the literature. Numerical results for nonlinear frequency and nonlinear displacements were presented and compared for various values of damping coefficients. To illustrate the value of this method, several applications were described. In addition to principal resonances, the present approach enables the user to obtain the higher harmonics resonances while taking into account a greater number of harmonics. Increasing the number of harmonics leads to increase the matrix decomposition and the second member calculus time. The time required to obtain a large section of the solution is also increased, but as a whole, the asymptotic numerical method takes less computational time than a classical incremental iterative algorithm [23].

Even if the second member time increases, it is negligible compared to the decomposition time. Then to reduce this computational time, one can use another linear solver which is welladapted to repeated right hand side problems [24], as with the asymptotic numerical method; reduce the number of steps by changing the path parameter definition [25] or use reduction methods to have to decompose a small size matrices [26,27]. These three approaches will be investigated in future works.

The presented work concerns only plates with moderate rotations, but complex models of plates and shells could be investigated [28] by taking into account large displacements and rotations. This has already been done in the static case $[29,30]$ by use of a particular formulation and finite element of type RAMM. The application of this approach in the dynamic case constitutes one of our perspectives.

\section{Appendix A}

The matrices $\overline{\boldsymbol{M}}, \overline{\mathbf{D}}$ and $\overline{\boldsymbol{B}}_{\boldsymbol{l}}$ used in Eq. (11) are symmetric and diagonal by blocs. For instance, with three harmonics, one has:
$\overline{\boldsymbol{C}}=\left[\begin{array}{ccccc}0 & 0 & 0 & 0 & 0 \\ 0 & 0 & C & 0 & 0 \\ 0 & C & 0 & 0 & 0 \\ 0 & 0 & 0 & 0 & 2 C \\ 0 & 0 & 0 & -2 C & 0\end{array}\right], \quad \bar{M}=\left[\begin{array}{ccccc}0 & 0 & 0 & 0 & 0 \\ 0 & M & 0 & 0 & 0 \\ 0 & 0 & M & 0 & 0 \\ 0 & 0 & 0 & 4 M & 0 \\ 0 & 0 & 0 & 0 & 4 M\end{array}\right]$

The matrix $\overline{\boldsymbol{B}}_{\boldsymbol{n l}}(\overline{\boldsymbol{U}})$ used in Eq. (10) corresponds to the product of two vectors $\boldsymbol{A}$ and $\boldsymbol{B}$, written in the form of series given in Eq. (10). For instance, with $H=3$, the product $\boldsymbol{A}(t) \times \boldsymbol{B}(t)$ results in:

$$
\begin{aligned}
\boldsymbol{A B}= & \boldsymbol{a}^{0} \boldsymbol{b}^{0}+\frac{1}{2}\left(\boldsymbol{a}_{c}^{1} \boldsymbol{b}_{c}^{1}+\boldsymbol{a}_{s}^{1} \boldsymbol{b}_{s}^{1}+\boldsymbol{a}_{c}^{2} \boldsymbol{b}_{c}^{2}+\boldsymbol{a}_{s}^{2} \boldsymbol{b}_{s}^{2}\right) \\
& +\left(\boldsymbol{a}^{0} \boldsymbol{b}_{c}^{1}+\boldsymbol{a}_{c}^{1} \boldsymbol{b}^{0}+\frac{1}{2}\left(\boldsymbol{a}_{c}^{1} \boldsymbol{b}_{c}^{2}+\boldsymbol{a}_{s}^{1} \boldsymbol{b}_{s}^{2}+\boldsymbol{a}_{c}^{2} \boldsymbol{b}_{c}^{1}+\boldsymbol{a}_{s}^{2} \boldsymbol{b}_{s}^{1}\right)\right) \cos (\omega t) \\
& +\left(\boldsymbol{a}^{0} \boldsymbol{b}_{s}^{1}+\boldsymbol{a}_{s}^{1} \boldsymbol{b}^{0}+\frac{1}{2}\left(\boldsymbol{a}_{c}^{1} \boldsymbol{b}_{s}^{2}-\boldsymbol{a}_{s}^{1} \boldsymbol{b}_{c}^{2}-\boldsymbol{a}_{c}^{2} \boldsymbol{b}_{s}^{1}+\boldsymbol{a}_{s}^{2} \boldsymbol{b}_{c}^{1}\right)\right) \sin (\omega t) \\
& +\left(\boldsymbol{a}^{0} \boldsymbol{b}_{c}^{2}+\boldsymbol{a}_{c}^{2} \boldsymbol{b}^{0}+\frac{1}{2}\left(\boldsymbol{a}_{c}^{1} \boldsymbol{b}_{c}^{1}-\boldsymbol{a}_{s}^{1} \boldsymbol{b}_{s}^{1}\right)\right) \cos (2 \omega t) \\
& +\left(\boldsymbol{a}^{0} \boldsymbol{b}_{s}^{2}+\boldsymbol{a}_{s}^{2} \boldsymbol{b}^{0}+\frac{1}{2}\left(\boldsymbol{a}_{c}^{1} \boldsymbol{b}_{s}^{1}+\boldsymbol{a}_{s}^{1} \boldsymbol{b}_{c}^{1}\right)\right) \sin (2 \omega t)
\end{aligned}
$$

The vector $\boldsymbol{B}$ can be put in as a factor, and the last equation can be written as follows:

$$
\left[\begin{array}{l}
A B^{0} \\
A B_{c}^{1} \\
A B_{s}^{1} \\
A B_{c}^{2} \\
A B_{s}^{2}
\end{array}\right]=\left[\begin{array}{ccccc}
a^{0} & \frac{1}{2} \boldsymbol{a}_{c}^{1} & \frac{1}{2} \boldsymbol{a}_{s}^{1} & \frac{1}{2} \boldsymbol{a}_{c}^{2} & \frac{1}{2} \boldsymbol{a}_{s}^{2} \\
\boldsymbol{a}_{c}^{1} & \boldsymbol{a}^{0}+\frac{1}{2} \boldsymbol{a}_{c}^{2} & \frac{1}{2} \boldsymbol{a}_{s}^{2} & \frac{1}{2} \boldsymbol{a}_{c}^{1} & \frac{1}{2} \boldsymbol{a}_{s}^{1} \\
\boldsymbol{a}_{s}^{1} & \frac{1}{2} \boldsymbol{a}_{s}^{2} & \boldsymbol{a}^{0}-\frac{1}{2} \boldsymbol{a}_{c}^{2} & -\frac{1}{2} \boldsymbol{a}_{s}^{1} & \frac{1}{2} \boldsymbol{a}_{c}^{1} \\
\boldsymbol{a}_{\boldsymbol{c}}^{2} & \frac{1}{2} \boldsymbol{a}_{c}^{1} & -\frac{1}{2} \boldsymbol{a}_{s}^{1} & \boldsymbol{a}^{0} & 0 \\
\boldsymbol{a}_{s}^{2} & \frac{1}{2} \boldsymbol{a}_{s}^{1} & \frac{1}{2} \boldsymbol{a}_{c}^{1} & 0 & \boldsymbol{a}^{0}
\end{array}\right] \times\left[\begin{array}{c}
\boldsymbol{b}^{0} \\
\boldsymbol{b}_{c}^{1} \\
\boldsymbol{b}_{s}^{1} \\
\boldsymbol{b}_{\boldsymbol{c}}^{2} \\
\boldsymbol{b}_{s}^{2}
\end{array}\right]
$$

Injecting (29) in (7) and applying harmonic balance, one finds, for $H=3$, the matrix $\overline{\boldsymbol{B}}_{\boldsymbol{n} l}(\overline{\boldsymbol{U}})$ as follows:

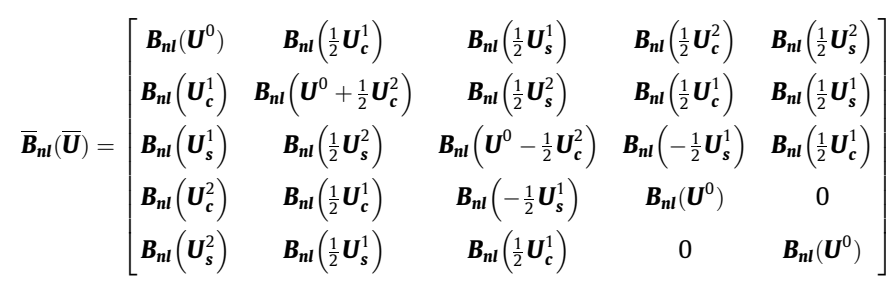

Note that $\overline{\boldsymbol{B}}_{\boldsymbol{n} \boldsymbol{l}}(\overline{\boldsymbol{U}})$ is not a symmetric matrix; its blocs are functions of the $\boldsymbol{B}_{\boldsymbol{n} \boldsymbol{I}}(\boldsymbol{U})$ defined in (3), it can be computed easily for any number of harmonics.

Here the unknown vector (10) is arranged according to harmonics blocs $i(i=0, H-1)$. In order to reduce the number of elements stored in the matrices, after discretization, the displacement vector is organized in the following form:

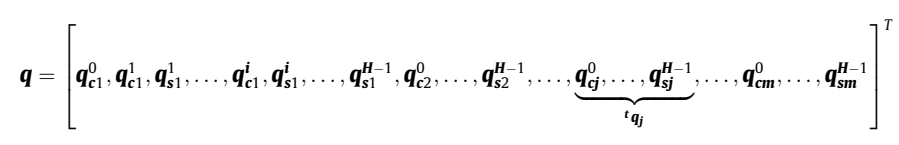

where $j$ is the degree of freedom $(j=1, m)$. This leads, with for instance 726 degrees of freedom and $H=3$, to decrease the number of terms saved in the tangent operator $\overline{\boldsymbol{K}}_{\boldsymbol{t}}$ by $80 \%$.

\section{References}

[1] Amabili M. Nonlinear vibrations of rectangular plates with different boundary conditions: theory and experiments. Comput Struct 2004;82:2587-605.

[2] Amabili M. Theory and experiments for large-amplitude vibrations of rectangular plates with geometric imperfections. J Sound Vibr 2006;291:539-65. 
[3] Amabili M. Nonlinear vibrations of circular cylindrical panels. J Sound Vibr 2005;281:509-35.

[4] Ganapathi M, Patel BP, Boisse P, Polit O. Flexural loss factor of sandwich and laminated composite beams using linear and nonlinear dynamic analysis. Composites Part B 1999;30:245-56.

[5] Daya EM, Azrar L, Potier-Ferry M. An amplitude equation for the non-linear vibration of viscoelastically damped sandwich beams. J Sound Vibr 2003;271(3):789-813.

[6] Boutyour EH, Daya EM, Potier-Ferry M. A harmonic balance method for the non-linear vibration of viscoelastic shell. Comptes Rendus Mécanique 2006;33:68-73.

[7] Touzé C, Amabili M. Nonlinear normal modes for damped geometrically nonlinear systems: application to reduced-order modelling of harmonically forced structures. J Sound Vibr 2006;298:958-81.

[8] Ribeiro P, Petyt M. Non-linear vibration of beams with internal resonance by the hierarchical finite element method. J Sound Vibr 1999;224(4):591-624

[9] Ribeiro P, Petyt M. Nonlinear vibration of plates by the hierarchical finite element and continuation methods. Int J Mech Sci 1999;41:437-59.

[10] Ribeiro P, Petyt M. Non-linear vibration of composite laminated plates by the hierarchical finite element method. Compos Struct 1999;46:197-208.

[11] Ribeiro P. Nonlinear vibrations of simply-supported plates by the $p$-version finite element method. Finite Element Anal Des 2005;41:911-24.

[12] Ribeiro P, Petyt M. Experimental detection of modal interaction in the nonlinear vibration of a hinged-hinged beam. J Sound Vibr 2004;277:943-54.

[13] Pérignon F. Vibrations forcées de structures, élastiques; nonlinéaire. Doctorat thesis, Université Aix-Marseille II, France; 2004

[14] Azrar L, Boutyour EH, Potier-Ferry M. Non-linear forced vibrations of plates by an asymptotic numerical method. J. Sound Vibr. 2002;252(4):657-74.

[15] Elhage-Hussein A, Potier-Ferry M, Damil N. A numerical continuation method based on Padé approximants. Int J Solid Struct 2000;37:6981-7001.

[16] Batoz J-L, Dhatt G. Modélisation des structures par éléments finis, vol 3: coques. Paris: Hermès; 1992.

[17] Cochelin B, Damil N, Potier-Ferry M. Asymptotic numerical methods and Padé approximants for non-linear elastic structures. Int J Numer Method Eng 1994;37:1187-213.
[18] Boutyour EH, Zahrouni H, Potier-Ferry M, Boudi M. Bifurcation points and bifurcated branches by Asymptotic Numerical Method and Padé approximants. Int J Numer Method Eng 2004;60:1987-2012.

[19] Mei C, Decha-Umphai K. A finite element method for non-linear forced vibrations of rectangular plates. Am Inst Aeronaut Astronaut J 1985;23:1104-10.

[20] Hsu CS. On the application of elliptic functions in non-linear forced oscillations. Quart Appl Math 1960;17:393-407.

[21] Mei C. Finite element displacement method for large amplitude free flexural vibrations of beams and plates. Comput Struct 1973;3:163-74.

[22] Ribeiro P. Periodic vibration of plates with large displacements. In: Proceedings of the 42nd AIAA/ASME/ASCE/AHS/ASC structures, structural dynamics, and material conference and exhibit, Seattle, WA, paper A01-25098; 2001.

[23] Abdoun F, Azrar L, Daya EM, Potier-Ferry M. Forced harmonic response of viscoelastic structures by an asymptotic numerical method. Comput Struct 2009;87:91-100

[24] Cadou J-M, Potier-Ferry M. A solver combining reduced basis and convergence acceleration with applications to non-linear elasticity. Commun Numer Method Eng. doi:10.1002/cnm.1246.

[25] Gervais J-J, Sadiky H. A new step length control for continuation with the asymptotic numerical method. IMA J Numer Anal 2002;22(2):207-29.

[26] Bathe KJ, Gracewski S. On nonlinear dynamic analysis using substructuring and mode superposition. J Comput Struct 1981;13:699-707.

[27] Rizzi S, Przekop A. System identification-guided basis selection for reducedorder nonlinear response analysis. J Sound Vibr 2008;315:467-85.

[28] Brank B, Mamouri S, Ibrahimbegovic A. Constrained finite rotations in dynamics of shells and Newmark implicit time-stepping schemes. Int J Eng Comput 2005;22:505-35.

[29] Zahrouni H, Cochelin B, Potier-Ferry M. Computing finite rotations of shells by an asymptotic-numerical method. Comput Method Appl Mech Eng 1999;175:71-85.

[30] Boutyour EH, Zahrouni H, Potier-Ferry M, Boudi M. Asymptotic-numerical method for buckling analysis of shell structures with large rotations. J Comput Appl Math 2004;168:77-85. 